fe zwischen 20 und 35 Jahren wird derjenige belegt, der eine Frau tötet, mit der er verheiratet ist oder als Lebensgefährte zusammen lebt. ${ }^{5}$

Wozu ein eigener Straftatbestand für die Tötung einer Frau? - mag man da als Ausländerin fragen. Das Töten eines Menschen, also Mord oder Totschlag, ist doch ohnehin in allen Gesetzbüchern schon mit den höchsten Strafen belegt! Wozu ist da noch eine Strafschärfung nötig? Die Costaricanerinnen antworten hier mit einer Gegenfrage: Kennen wir in Deutschland nicht auch die Fälle, in denen der Täter seine Ehefrau und vielleicht auch noch die Kinder umbringt, und dann aber vor Gericht unter Hinweis auf die überaus emotionale psychische Ausnahmesituation, in der er sich unmittelbar vor und während der Tatbegehung befunden hatte (hat doch das Opfer mit Trennung gedroht!) eine starke Strafmilderung erhält?! Dem würde der Gedanke des Femizids eine Strafschärfung entgegensetzen, so dass am Ende doch eine angemessenere Strafe stehen könnte.

Den feministisch engagierten Costaricanerinnen geht aber die Gesetzesfassung der „Ley de penalización“ nicht weit genug. Zunächst wird kritisiert, dass das Gesetz nur die Tötung von (Ehe-)Partnern erfasst und nicht auch Taten in beendeten Beziehungen. Wichtiger scheint aber, dass bei der Gesetzesfassung nicht zum Ausdruck kommt, dass ein Femizid die besonderen Umstände der Tötung der Frau beschreibt, dass die Frau gerade deswegen getötet wurde, weil sie eine Frau ist. Es handelt sich bei dem Femizid um einen Ausdruck der ungleichen Macht- und Herrschaftsverhältnisse zwischen den Geschlechtern und die maximale Ausprägung der häuslichen Gewalt. Besonders wird kritisiert, dass diese nur mittelmäßige
Gesetzgebung dann in der Praxis noch nicht einmal mit dem angemessenen Nachdruck umgesetzt werde.

Hier schließt sich der Kreis: Jedes Gesetz ist nur so gut oder schlecht, wie es angewendet wird. Angesichts der Zahlen in Deutschland würde es uns gut anstehen, den Impuls aus Mittelamerika aufzunehmen und eine Diskussion in Richtung der Schaffung eines eigenen Straftatbestands Femizid zu führen: Im Jahr 2011 wurden in Deutschland 154 Frauen von ihrem Mann, Lebensgefährten oder Ex-Partner umgebracht ${ }^{6}$, was uns zu einer Reaktion drängen muss. Von der Einführung des neuen Straftatbestands wäre nicht nur zu erwarten, dass sie voraussichtlich die Chance einer angemessenen Bestrafung der Täter erhöhen und damit general- und spezialpräventiv wirken würde. Hinzu kommt der nicht zu unterschätzende Effekt, den die Diskussion in der (Fach-)Öffentlichkeit um die Schaffung eines neuen Gesetzes zwangsläufig mit sich bringt, indem die hinter dem diskutierten Gesetzentwurf stehende Realität für die Gesellschaft offengelegt und verdeutlicht wird. Gleichzeitig muss aber unbedingt die Aus- und Fortbildung der Rechtsanwender/innen zu der Thematik „Gewalt gegen Frauen“ verstärkt werden, um der Praxis mehr Rechtssicherheit zu geben und um zu erreichen, dass sie - anders als in Costa Rica - die Intentionen und Möglichkeiten des Gesetzes ausschöpft und nicht an ihnen vorbei handelt.

5 Zum Vergleich: In Art. 111 des costaricanischen Strafgesetzbuches ist Totschlag mit zwischen 12 und 18 Jahren Freiheitsstrafe bewehrt, Art. 112 sieht für verschärfte Fälle (teilweise vergleichbar mit dem deutschen „Mord“) Freiheitsstrafen zwischen 20 und 35 Jahren vor.

6 Zitiert nach Süddeutsche Zeitung vom 23.5.2012, <http://www.sueddeutsche.de/panorama/frauenmorde-sind-haeufig-beziehungstaten-toedliche-zweisamkeit-1.1365223> (Zugriff: 17.10.2012).

\title{
69. Deutscher Juristentag in München, 17. bis 21. September 2012
}

\section{Anke Gimbal \\ Geschäftsführerin des djb, Berlin}

Vom 17. bis 21. September 2012 tagte der 69. Deutsche Juristentag (djt) mit ca. 2.000 Teilnehmerinnen und Teilnehmern in München. Kurz vor Beginn des Oktoberfestes waren die Hotelpreise - in München ohnehin hoch - astronomisch, was sicher die oder den ein/e/n oder andere/n von der Teilnahme abgehalten hat. Der „Oktoberfestabend“, zu dem der Juristentag zusammen mit dem Bundesministerium der Justiz für den Dienstag im Münchner Hofbräuhaus eingeladen hatte, fand jedoch großen Zuspruch. Anstelle des schwarzen Anzugs trug man Dirndl oder Lederhose. Statt Prosecco und Sushi, Tomate-Mozzarella oder Ähnlichem gab es Maßkrüge mit hauseigenem Bier, Brotzeitbrettl und ein Haxn-Buffet.

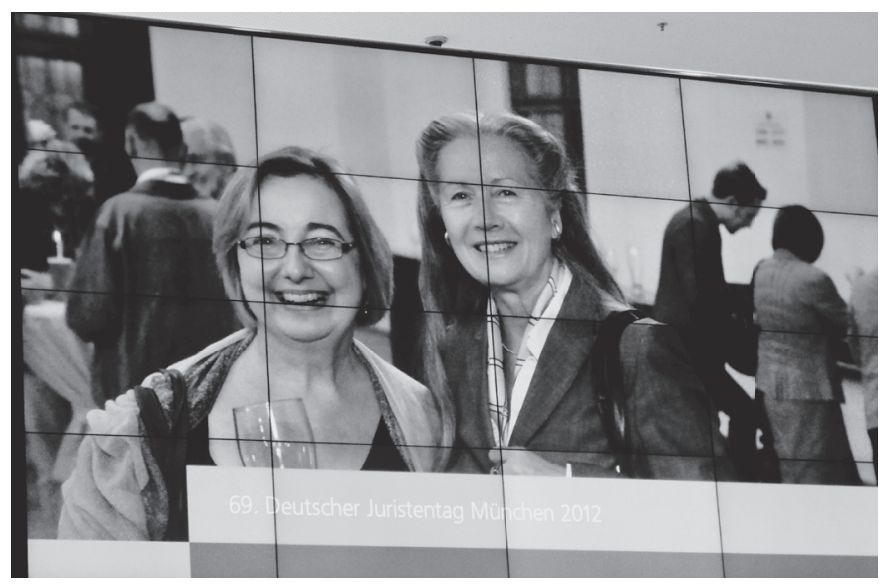

- Präsidentin Ramona Pisal (rechts) mit Rechtsanwältin Petra Heinicke, 1. Vorsitzende des Münchener Anwaltvereins, djb-Bundesvorstandsmitglied von 1993 bis 1995. (Foto: CD, djb) 


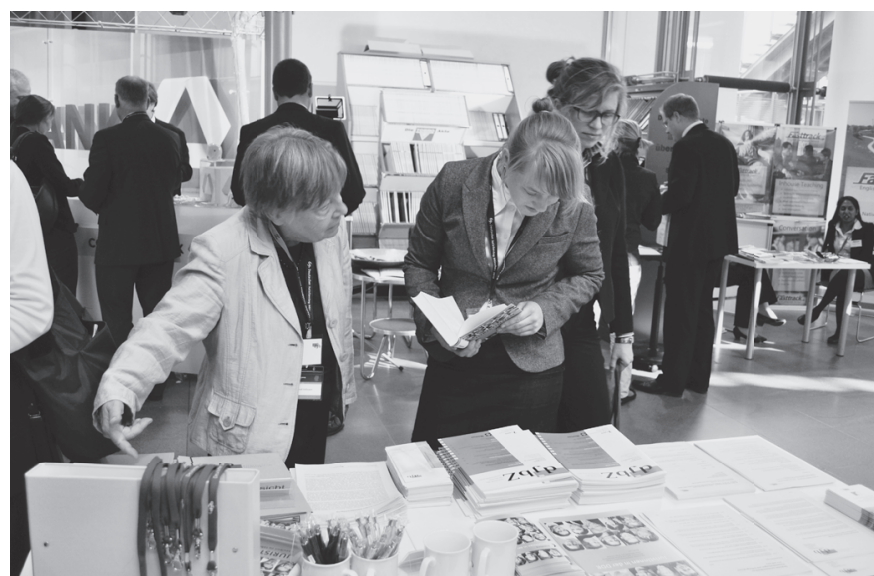

$\Delta$ Gertrud Hofmann, Richterin am OLG a.D., Bundesvorstandsmitglied von 1979 bis 1993, Ehrenvorsitzende der RG München im djb lädt djt-Besucher/ innen ein, sich am Ausstellungsstand des djb mit dem Thema Gleichstellung von Frauen auseinanderzusetzen. (Foto: $C D, \mathrm{djb}$ )

Präsident Prof. Dr. Martin Henssler eröffnete und leitete den Juristentag 2012 zum dritten und letzten Mal. Zu seinem Nachfolger und damit zugleich zum Präsidenten des 70. Juristentags in Hannover 2014 bestimmte die Ständige Deputation den bisherigen Schatzmeister, Rechtsanwalt Prof. Dr. Thomas Mayen aus Bonn. Prof. Dr. Astrid Stadler aus Konstanz wurde zur Stellvertretenden Vorsitzenden gewählt. Die bisherige Stellvertretende Vorsitzende, Prof. Dr. Ingeborg Schwenzer, Basel, schied nach zwei sechsjährigen Amtszeiten turnusmäßig aus der Ständigen Deputation aus. Neu in die Ständige Deputation gewählt wurden Prof. Dr. Nina Dethloff, Bonn, und Rechtsanwältin sowie Richterin des Berliner VerfGH Anke Müller-Jacobsen. Nach wie vor überwiegen bei den Teilnehmern des Deutschen Juristentages, den Mitgliedern der Ständigen Deputation (Männerquote von $80 \%$ ), den Gutachtern und Referenten der Abteilungen (Männerquote von 84\%) die Juristen.

Der djb war wie in den letzten Jahren mit einem Stand vertreten. Die djb-Mitarbeiterinnen Katrin Lange, zuständig für das djb-Projekt „Aktionärinnen fordern Gleichberechtigung“, und Cornelia Dahlitz wurden dankenswerterweise unterstützt durch die Mitglieder der Regionalgruppe München/Südbayern Gertrud Hofmann und Sabine Lutzenberger. Neben den üblichen Informationsangeboten gaben die djb-Mitarbeiterinnen dieses Jahr anlässlich der zu erwartenden Diskussionen über die Frauenquote in der Wirtschaftsabteilung zusätzlich umfassend Auskunft über das Projekt „Aktionärinnen fordern Gleichberechtigung“, warben für die bevorstehende Präsentation der Projektstudie 2012, die Hauptversammlungsbesuche 2013 und verteilten die beiden djb-Studien der Jahre 2011 und 2010 .

\section{djb-Empfang im Münchener Justizpalast}

Am Mittwochabend, 19. September 2012, fand - organisiert durch Dr. Monika Zumstein, Vorsitzende der Regionalgruppe München/Südbayern im djb - im Justizpalast der traditionelle Abendempfang des djb anlässlich eines djt statt. Präsiden- tin Ramona Pisal begrüßte die ca. 250 Gäste. Der Präsident des djt, Prof. Dr. Martin Henssler, sprach ein Grußwort und dankte nicht nur für die Einladung zum Empfang, der er gerne gefolgt sei, sondern überhaupt für die Bereicherung des djt durch den djb und dessen Mitglieder. Auch viele Münchener djb-Mitglieder waren der Einladung gefolgt.

Dieses Jahr war der Empfang mit einem besonderen Highlight versehen, der Verleihung des „Preis Frauen Europas 2012 - Deutschland“ durch die Europäische Bewegung Deutschland. Die Juristin Jasmina Prpić, LL.M., Freiburg ist die diesjährige Preisträgerin. Prpić wurde 1954 in Banja Luka (Bosnien und Herzegowina) geboren. Der ausbrechende Krieg auf dem Balkan zwang die Richterin erst zur Aufgabe ihres Amtes und 1992 zur Flucht nach Deutschland. Als Flüchtling ohne Deutschkenntnisse wäre die Wiederaufnahme ihrer juristischen Tätigkeit der einfachste Schritt zur Überwindung ihres Traumas gewesen. Doch der blieb ihr verwehrt. Ihre Ausbildung wurde nicht anerkannt, weil sie ihren Abschluss nicht in einem EU-Mitgliedstaat erworben hatte. Sie musste in Deutschland ein weiteres Mal Jura studieren. Bei einem Aufenthalt im Kosovo für medica mondiale e.V., bei dem sie Opfer sexualisierter Gewalt als mögliche Zeuginnen vor dem Haager Kriegsverbrechertribunal begleitete, wurde ihr bewusst, wie sehr Frauen und Frauenorganisationen juristische Hilfe benötigen. Daher gründete sie 2007 mit elf weiteren Juristinnen den Verein „Anwältinnen ohne Grenzen e.V.“, dessen Vorsitzende sie seitdem ist. Der Verein kämpft für die Förderung und Durchsetzung der Menschenrechte von Frauen und den Abbau jeglicher Form von Ungleichbehandlung oder Diskriminierung.

Im Anschluss an die Laudatio durch Staatssekretärin Gudrun Kopp, Bundesministerium für wirtschaftliche Zusammenarbeit und Entwicklung, steckte Prof. Gudrun Schmidt-Kärner, Präsidentin des „Preis Frauen Europas - Deutschland“, der Preisträgerin die Nadel an. Der Abend schloss ganz zünftig in der Oktoberfestzeit mit Brezn, Leberkäs, Bier und vielen Gesprächen.

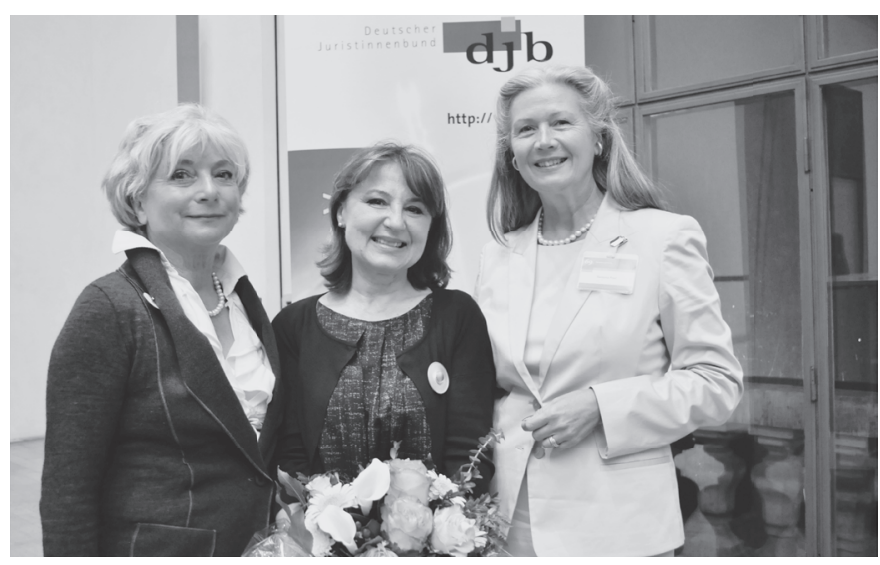

A Preisträgerin Jasmina Prpić, LL.M. (Mitte), mit djb-Präsidentin Ramona Pisal (rechts) und Dr. Monika Zumstein, Akademische Direktorin a.D., Bundesvorstandsmitglied von 1985 bis 1991, Redakteurin der ,aktuellen informationen“ des djb von 1991 bis 2000, Vorsitzende der RG München/Südbayern im djb. (Foto: Katrin Lange, djb) 


\section{Abteilung Wirtschaftsrecht: Frauenquote}

Mittwoch und Donnerstag fanden wie üblich die Abteilungssitzungen statt, an deren Ende Beschlüsse und Empfehlungen des djt standen. djb-Mitglieder waren in allen Abteilungen vertreten, doch die Abteilung Wirtschaftsrecht fand besondere Aufmerksamkeit. Unter dem Titel „Staatliche und halbstaatliche Eingriffe in die Unternehmensführung " wurde dort unter anderem über die Einführung einer gesetzlichen Frauenquote für die Vorstände und Aufsichtsräte der börsennotierten Unternehmen diskutiert. EU-Kommissionsvizepräsidentin Viviane Reding hatte in ihrer Festrede anlässlich der Eröffnung des djt am Dienstag betont, dass eine Frauenquote kommen werde. Sie sei sicher, dass es auch in Deutschland bis 2015 eine Quotenregelung geben werde. Denn elf EU-Mitgliedstaaten haben bereits Quotenregelungen in der einen oder anderen Form, so Reding.

352 Anmeldungen lagen für die Abteilung Wirtschaftsrecht vor. Ca. 80 Mitglieder waren bei der Abstimmung am Donnerstagnachmittag zugegen. Erwartungsgemäß lehnten die überwiegend im Gesellschaftsrecht tätigen Juristen eine Frauenquote für Aufsichtsräte und Vorstände börsennotierter Unternehmen als unberechtigte Indienstnahme der Unternehmen für gesellschaftspolitische Anliegen ab. Schon der Gutachter Prof. Dr. Mathias Habersack, München, hatte sich in seinem vorab veröffentlichten Gutachten gegen eine Quote ausgesprochen. In den Thesen zur Diskussion, die insbesondere für jene auslagen, die das umfangreiche Gutachten nicht gelesen hatten, hieß es:

„2. Indienstnahme der AG für gesellschaftspolitische Anliegen Zur Frage einer Frauenquote

(3) Die Aktiengesellschaft sieht sich der Gefahr einer zunehmenden Indienstnahme für gesellschaftspolitische Anliegen ausgesetzt. Neben der - immerhin einen Corporate-Governance-Bezug aufweisenden - unternehmerischen Mitbestimmung ist vor allem die Forderung nach stärkerer Repräsentanz von Frauen in den Gesellschaftsorganen zu nennen.

(4) Eine gesetzliche Frauenquote wäre, auch wenn sie auf den Aufsichtsrat beschränkt würde, ein aktienrechtlicher Fremdkörper und würde die durch die mitbestimmungsrechtlichen Gesetze und durch $\S \S 100$ Abs. 5, 107 Abs. 4 AktG ohnehin schon erheblich eingeschränkte Wahlfreiheit der Aktionäre in bedenklicher Weise weiter einengen. Der Kreis der in eine gesetzliche Frauenquote einzubeziehenden Gesellschaften ließe sich nicht ohne Inkaufnahme von Wertungswidersprüchen begrenzen; namentlich fehlt es an einem sachlichen Zusammenhang mit der Börsennotierung und der Mitbestimmung der Gesellschaft. Wollte man mitbestimmte Gesellschaften einer gesetzlichen Frauenquote unterstellen, wäre die Quote auf den Gesamtaufsichtsrat zu beziehen." (<http://www.djt.de/fileadmin/downloads/69/120809_djt_69_thesen_web.pdf>, Zugriff: 5.10.2012)

Vertreterinnen des djb forderten dagegen in ihren Diskussionsbeiträgen mit Blick auf die Ergebnisse des djb-Projektes „Aktionärinnen fordern Gleichberechtigung" (http://www. djb.de/Projekt_HV/) eine verbindliche Quote zur Sicherung gerechter Teilhabe der Frauen an den Führungspositionen der
Wirtschaft. Prof. Dr. Glock, Vorsitzende des djb-Landesverbands Berlin, vertrat die djb-Kommission Arbeits-, Gleichstellungs- und Wirtschaftsrecht und äußerte in ihrem Diskussionsbeitrag u.a.: „Viertens ist die Behauptung des Gutachters nicht nachvollziehbar, dass die Quotenregelung ein bedenklicher Fremdkörper im Aktienrecht sei, denn auch die Vorschriften des Aktiengesetzes stehen in der gesamtpolitischen Ordnung des Grundgesetzes. Es ist keineswegs eine Tatsache, dass Frauen bis heute noch Schwierigkeiten haben, Karriere und Familie zu vereinen und deshalb eine Entscheidung häufig gegen die Karriere treffen. Der Gutachter hat hier insbesondere außer Acht gelassen, dass bereits mehr als die Hälfte aller akademisch ausgebildeten Frauen keine Familie haben und infolgedessen sehr wohl in der Lage sind, den vom Gutachter beschriebenen Karriereweg mit einer wie er meint typischerweise verbundenen Belastung einer 60-80-Stundenwoche zu meistern." Der Redebeitrag von Präsidentin Ramona Pisal ist im Wortlaut hier abgedruckt. Der djb fordert die Quote per Gesetz. Selbstverpflichtungen der Unternehmen sind weitgehend wirkungslos geblieben, denn freiwillig wird nicht geteilt. Trotz der Ablehnung der Frauenquote durch die djt-Abteilung Wirtschaftsrecht führte die intensive Debatte und Auseinandersetzung mit den Argumenten des djb dazu, dass die Abteilung in Abänderung der Beschlussvorlage die Bemühungen des Corporate Governance Kodex um eine verstärkte Beteiligung von Frauen in Führungsgremien ausdrücklich begrüßte und auf Antrag der djb-Vertreterinnen empfahl, die in anderen europäischen Ländern bereits bestehenden Quotenregelungen näher zu untersuchen. Die Abteilungsbeschlüsse zur Frauenquote im Einzelnen:

„3. Frauenquote (dafür:dagegen:enthalten)

a) Die Bemühungen des Kodex um eine verstärkte Beteiligung von Frauen in Führungsgremien einschließlich der selbst gesetzten Quote für den Aufsichtsrat sind zu begrüßen. angenommen 71:2:6

b) Weder der deutsche noch der europäische Gesetzgeber sollte eine Frauenquote für Vorstände anordnen. angenommen 58:15:3

c) Weder der deutsche noch der europäische Gesetzgeber sollte eine Frauenquote für Aufsichtsräte anordnen. angenommen 62:16:2

d) Weder der deutsche noch der europäische Gesetzgeber sollte eine flexible, selbst gesetzte Frauenquote für Aufsichtsräte anordnen. angenommen 54:21:5

e) Die Auswirkungen der in anderen europäischen Ländern bereits bestehenden gesetzlichen Regelungen zur Frauenquote sollten näher untersucht werden. angenommen 73:3:11 (<http://www.djt.de/fileadmin/downloads/69/120921_djt_69_ beschluesse_web_rz.pdf $\rangle$, Zugriff: 5.10.2012)

Der djb wird jedenfalls auch 2013 das Projekt „Aktionärinnen fordern Gleichberechtigung “ fortsetzen, ca. 75 Hauptversammlungen börsennotierter Unternehmen besuchen und Fragen zur Besetzung der Führungspositionen in den Unternehmen mit Frauen stellen. Handelsgesetzbuch, Wertpapierhandelsgesetz und Deutscher Corporate Governance Kodex legen 
den Unternehmen Berichtspflichten auf. Wir fordern Transparenz und uns interessiert insbesondere, welche Strategien verfolgt werden, um Karrieren von Frauen im Unternehmen bis in die Chefetagen zu fördern. Vor allem: Gibt es solche Strategien überhaupt? Wie gesagt - Karriere ist kein Selbstläufer. „Aber Eigentum verpflichtet. Das weiß jeder. Comply or explain.“, so das Schlusswort von Präsidentin Ramona Pisal an die Abteilung.

\section{Wortbeitrag von Ramona Pisal}

Ich melde mich hier als Präsidentin des Deutschen Juristinnenbundes (djb) zu Wort. Mein Beitrag wird ein rechtspolitischer sein. Der djb ist ein Verband von Juristinnen, Volkswirtinnen und Betriebswirtinnen, nicht berufsständisch, der sich seit mehr als 60 Jahren für die Durchsetzung der tatsächlichen Gleichberechtigung der Frauen in allen Bereichen des gesellschaftlichen Lebens einsetzt. Vor diesem Hintergrund freuen wir uns besonders, dass in dieser Abteilung als staatlicher Eingriff auch die Einführung einer gesetzlichen Quote für Frauen in Führungspositionen der Unternehmen sowie die Empfehlungen des DCGK als halbstaatlicher Eingriff angesprochen werden.

Insbesondere drei Aussagen des Gutachters möchte ich hier ansprechen und dazu Stellung nehmen:

1. Der Gutachter widmet dem Thema „Frauenquote“ ein eigenes Kapitel unter III.2. und schreibt: „dem deutschen Gesetzgeber ist dringend anzuraten, von einer gesetzlichen Regelung abzusehen“ - und behauptet dann: „Er (der deutsche Gesetzgeber) kann dies guten Gewissens tun, nachdem die DAX30-Gesellschaften ganz überwiegend den Empfehlungen in 5.4.1 folgen und sie sich zudem Ende März 2011 öffentlich dazu verpflichtet haben, sich jeweils eine Frauenquote aufzuerlegen und diese bis Mitte 2013 umzusetzen." (Gutachten Seite 40). Das Gutachten verschweigt hier zunächst, dass die Selbstverpflichtungen der DAX30-Unternehmen sich in keinem Fall auf die Aufsichtsräte und die Vorstände beziehen. Im Übrigen schweigt es leider zu den Erkenntnisgrundlagen und Erfahrungswerten, auf denen diese Beschwichtigung und der sicher gerne gehörte Rat zum Nichtstun beruhen.

Wir haben aber solche Erkenntnisse, und sie belegen das Gegenteil: die DAX30-Gesellschaften folgen den Empfehlungen in Nr. 5.4.1 des DCGK ganz überwiegend nicht.

Im Rahmen eines vom Bundesministerium für Familie, Senioren, Frauen und Jugend geförderten Projekts besuchen wir 76 Hauptversammlungen großer Aktiengesellschaften, darunter die Dax30-Unternehmen. Unter dem Motto „Aktionärinnen fordern Gleichberechtigung “ hinterfragen wir die Befolgung der Empfehlungen des Kodex und die Umsetzung der Selbstverpflichtungen.

Die Auswertung der diesjährigen Aktion wird gerade abgeschlossen. Hierzu wird es eine Veröffentlichung geben, die wir am 28. November 2012 im Allianz Forum in Berlin vorstellen werden.

Demzufolge gibt es eine Steigerung des Frauenanteils in den von uns besuchten 76 Unternehmen, darunter die DAX30, in den Aufsichtsräten und den Vorständen, die bei den DAX30 deutlicher ausfällt als bei den anderen 46 Unternehmen.

Von Anteilseignerseite werden derzeit 38 Frauen in die Aufsichtsräte der DAX 30 entsandt, das sind knapp 15 Prozent; die übrigen 218 Mandate bekleiden Männer. In den Vorständen der DAX30 sind weniger als acht Prozent Frauen, konkret gesagt: 14 Frauen gegenüber 177 Männern, deren Quote mehr als 92 Prozent beträgt. In den übrigen Unternehmen sind es lediglich knapp vier Prozent.

Nur sechs der DAX30-Unternehmen gaben an, konkrete Maßnahmen zu ergreifen, um für die nächste Wahl zum Aufsichtsrat auch Frauen vorschlagen zu können.

Nur sechs der DAX30-Unternehmen gaben an, Frauen für Vorstandspositionen zu entwickeln, die anderen 70 Unternehmen gaben hierzu mehr oder weniger keine Antwort

Jede fünfte der Neu- oder Wiederwahlen in diesem Jahr entfiel auf Frauen.

Nur ein Drittel der Unternehmen nimmt Personalberatung in Anspruch und fragt gezielt nach Frauen. Ungefragt teilte ein Drittel der Unternehmen aber mit, beinahe ausschließlich über eigene Netzwerke nach neuen Mitgliedern für den Aufsichtsrat zu schauen.

Bei diesem Trend will ich es hier belassen. Die Einzelheiten werden Sie bei Interesse später unserer Studie entnehmen können.

Ausgehend von den diesjährigen Zuwachsquoten würde das Erreichen des von uns geforderten Frauenanteils von 40 Prozent noch einmal zwanzig Jahre dauern für den Vorstand und fast zehn Jahre für die Aufsichtsräte.

Trotzdem geben die Unternehmen uneingeschränkte Entsprechenserklärungen $\mathrm{ab}$.

2. Monokausal betont der Gutachter, dass „Frauen in der Vergangenheit erhebliche Schwierigkeiten hatten und noch heute haben, Karriere und Familie zu vereinbaren, und deshalb häufig auch aus aussichtsreichster Position heraus eine Entscheidung gegen ihre Karriere treffen, etwa weil sich die mit dem kontinuierlichen Beschreiten des Karrierewegs typischerweise verbundenen Belastungen - 60-80-Stundenwoche, Auslandseinsätze - als ganz und gar unvereinbar mit familiären Belangen erweisen“ (S. 36/37). Ich würde gerne wissen, wie das z.B. in Ihren Ohren, Frau Weber-Rey, klingt.

Hier bemüht der Gutachter mit bedenklicher Ausschließlichkeit ein Rollenstereotyp als Erklärungsmodell, mit dem sich weder Frauen noch deren Partner und Partnerinnen identifizieren werden, sieht er doch mit diesem Satz die „familiären Belange“ ganz und gar als Frauensache an. So formuliert würde Frauen mit Familie, wobei noch zu definieren bliebe, was darunter zu verstehen ist, der Weg in die obersten Etagen der Wirtschaft auf ewig verschlossen bleiben. Während die meisten männlichen Führungskräfte der deutschen Wirtschaft Familie und Kinder haben, das kontinuierliche Beschreiten des Karrierewegs samt den typischerweise damit verbundenen Belastungen - 60-80-Stundenwoche, Auslandseinsätze - sich für Männer als ganz und gar vereinbar mit familiären Belangen erweist? 
Davon abgesehen: 40 Prozent der hoch qualifizierten Frauen sind kinderlos. Für sie gilt die ganz und gare Unvereinbarkeit nicht. Trotzdem finden wir sie nicht in den Chefetagen.

3. Der Gutachter hält eine Quotenregelung für einen „bedenklichen Fremdkörper“ im Aktienrecht. Ihm ist deutlich unwohl bei dem Gedanken, „privatrechtlich verfasste Organisationen soll(t)en für die Bewältigung eines - berechtigten gesellschaftspolitischen Anliegens in die Pflicht genommen werden, ..." (S. 36).

Nach dem Recht der reinen Aktienlehre mag das so sein. Aber rechtspolitisch muss man doch sehen: ohne gesetzliche Vorgabe ändert sich nichts.

Mit dem Zusatz zu Artikel 3 Absatz 2 GG ist ein klarer Verfassungsauftrag zu aktivem Handeln formuliert worden: „Der Staat fördert die tatsächliche Durchsetzung der Gleichberechtigung von Frauen und Männern und wirkt auf die Beseitigung bestehender Nachteile hin. " So steht es im Grundgesetz und seitdem sind erneut fast zwei Jahrzehnte ohne nennenswerten Fortschritt vergangen. Ich glaube, wir brauchen niemanden zu ermutigen, nicht zu entsprechen und abzuweichen.

Die Selbstverpflichtung der deutschen Wirtschaft, eine Vereinbarung mit der Regierung, ist jetzt bereits 11 Jahre alt, ohne dass sie eingelöst worden wäre.

Veränderungen ergeben sich erst im durchaus überschaubaren Zeitraum seit 2009, wozu auch der Gutachter konzidiert: „dabei soll nicht verkannt werden, dass die Gesellschaften bei alledem unter dem Eindruck der seitens der Politik unverblümt angedrohten gesetzlichen Regelung gehandelt haben" (S. 40).

Sicher wäre ein höherer Frauenanteil in den Führungsetagen auch im Interesse der Unternehmen, denn der demografische Wandel und der Fachkräftemangel sollten schon Grund genug sein, die gesamte arbeitsfähige Bevölkerung in den Blick zu nehmen und deren Potenziale zu nutzen, nicht nur zur Hälfte.

Und es mag auch sein, dass es sich sogar rechnen würde für die Unternehmen, denn namhafte Wirtschaftsberatungen kommen zu dem Ergebnis, dass ein hoher Frauenanteil im Unternehmensmanagement einen höheren Gewinn generiert. Näheres dazu z. B. in aktuellen Studien von McKinsey, Ernst $\&$ Young und Catalyst.

Ich bin weder Aktienrechtlerin noch Wirtschaftsfrau und als Juristin sind mir diese Anreize auch gar nicht so wichtig. Für mich stehen andere Aspekte im Vordergrund: Mir geht es um Rechtstreue, Rechtlichkeit und letztlich Gerechtigkeit, so altmodisch das in manchen Ohren klingen mag.

Junge Menschen, ihre Familien und auch die Steuerzahler investieren viel Zeit und Geld in Ausbildung. Es ist eine ungeheure Verschwendung, den Erfolg dieser Investitionen nur zur Hälfte zu nutzen und gleichzeitig eine grobe Ungerechtigkeit, die Karrierewege je nach Geschlecht zu eröffnen oder zu versperren. Das hat sich unsere Verfassung anders gedacht und mit dem Zusatz zu Artikel 3 Absatz 2 nochmals verdeutlicht.

Es ist die Aufgabe des Gesetzgebers, die Wirklichkeit in den Blick zu nehmen und mit geeigneten Maßnahmen auf die
Herstellung verfassungs- und rechtsgemäßer Zustände hinzuwirken, wenn sie sich nicht von selbst ergeben. Nichts spricht dafür - entgegen dem Gutachten -, dass der Gesetzgeber hier auf solche Maßnahmen verzichten kann, denn in mehr als 60 Jahren hat sich insoweit nichts von selbst ergeben. Und das wird es auch nicht, denn es geht um Macht, um Teilhabe an Gestaltungsmöglichkeiten und Einfluss; freiwillig wird hier nicht geteilt.

Frauen wollen sich in allen gesellschaftlichen Bereichen angemessen, d.h. eigentlich zur Hälfte, repräsentiert sehen. Das kann man ihnen in einem Rechtsstaat nicht schlicht verweigern mit dem lapidaren Hinweis, man halte den Weg über eine gesetzliche Regelung nicht für den richtigen, wenn man andererseits jeden Nachweis anderer erfolgversprechender Mittel schuldig bleibt. Eines demokratisch verfassten Rechtsstaates ist das nicht würdig.

Dass eine gesetzliche Quotenregelung verfassungskonform ausgestaltet werden kann, sogar für den Vorstand, bejaht auch Herr Prof. Seibert für das Bundesministerium der Justiz; beruhigen, diesmal zu Recht, könnte man den Gesetzgeber auch mit Blick auf das insoweit eindeutige Votum des ehemaligen Präsidenten des Bundesverfassungsgerichtes, Herrn Professor Papier.

Das Unbehagen gegenüber einem „aktienrechtlichen Fremdkörper" müsste den Gesetzgeber nicht beunruhigen, denn zahlreiche derartiger „Fremdkörper“ sind in der Vergangenheit bereits in das Aktienrecht inkorporiert worden, wenn man die Diskussionen z. B. zur materiellen Kontrolle und des Gesetzes zur Überprüfung der Angemessenheit der Vorstandsvergütungen verfolgt. Da wird auch ein weiterer „Fremdkörper" keinen besonderen Schaden anrichten.

Fraglos tragen Unternehmen eine soziale Verantwortung, und der Gutachter erwähnt den aus Sicht des deutschen Gesellschaftsrechtes bemerkenswerten Umstand, „dass hierüber in anglo-amerikanisch geprägten Rechtsordnungen lebhaft nachgedacht wird und diese Überlegungen nachgerade in eine Corporate Social-Responsibility-Bewegung und auf europäischer Ebene bereits im Jahr 2001 in ein entsprechendes Grünbuch gemündet sind“ (Seite 15).

Auch er sieht, dass „die Erfahrungen mit der Finanz- und Wirtschaftskrise den nicht wenigen Befürwortern einer vorrangigen oder gar alleinigen Ausrichtung des Vorstandshandelns am Aktionärsinteresse (vor allem am Marktwert der Beteiligung) einigen Wind aus den Segeln genommen und umgekehrt die Corporate Social Responsibility-Debatte beflügelt haben dürften (S. 16), stellt aber fest, „dass der Vorstand einer AG zur Förderung sozialer und vergleichbarer Belange im Grundsatz befugt, indes nicht verpflichtet ist".

Aber Eigentum verpflichtet doch. Das weiß jeder, nicht nur wir Juristen.

Comply or explain.

Oder sollen wir wirklich anfangen, die Entsprechenserklärungen und die Beschlüsse der Hauptversammlung anzufechten? 